\title{
Multi-frequency radio observations of BAL quasar $1045+352$
}

\section{Magdalena Kunert-Bajraszewska*}

Toruń Centre for Astronomy, N. Copernicus University, 87-100 Toruń, Poland

E-mail: magda@astro.uni.torun.pl

\section{Andrzej Marecki}

Toruń Centre for Astronomy, N. Copernicus University, 87-100 Toruń, Poland

E-mail: amr@astro.uni.torun.pl

Multi-frequency 1.7, 5 and 8.4-GHz VLBA observations of a radio-loud broad absorption line (BAL) quasar 1045+352 are presented. It is a young compact steep spectrum (CSS) object and its asymmetric, two-sided morphology on a scale of several hundred parsecs, extending in two different directions, may suggest intermittent activity. The young age and unusual morphology of $1045+352$ are arguments in favour of an evolution scenario for BAL quasars, in which the BAL features appear at a very early stage of their evolution.

8th European VLBI Network Symposium

September 26-29, 2006

Toruń, Poland

${ }^{*}$ Speaker. 


\section{Introduction}

Approximately $10 \%$ of optically selected radio-quiet quasars display broad absorption lines (BALs) in the blue wings of the high ionization resonant lines (C IV $1549 \AA$ - high-ionization BAL (HiBAL) quasars), caused by the outflow of gas with velocities up to $\sim 0.2 c$ [9]. $10 \%$ of them also show absorption troughs in low ionization lines (Mg II $2800 \AA$ - low-ionization BAL (LoBAL) quasars). Evidence has accumulated from optically selected BAL quasars, in favour of an orientation hypothesis to explain their nature. According to this hypothesis, BAL regions exist in both BAL and non-BAL quasars, and that BAL quasars are normal quasars seen along a particular line of sight, e.g. a line of sight skimming the edge of the accretion disk or torus [21]. This view has been challenged by the discovery of the existence of a large population of radio-loud BAL quasars [3, 2, 15]. Most of these quasars tend to be compact in the radio domain with either a flat or steep radio spectrum. It follows that those belonging to the latter class seem to be related to Gigahertz-Peaked Spectrum (GPS) and Compact Steep Spectrum (CSS) sources. This variety of spectral indices suggests a wide range of orientations, contrary to the interpretation favoured for optically selected quasars.

The radio morphology of BAL quasars is important because it can serve as an inclination indicator of BALs, and therefore yields a direct test of the orientation model. However, information about the radio structure of BAL quasars is still very limited. Prior to 2006, only three BAL quasars, FIRST J101614.3+520916 [7], PKS 1004+13 [24] and LBQS 1138-0126 [4] were known to have a double-lobed FR II radio morphology on kiloparsec scales, although this interpretation is doubtful for PKS 1004+13 [6]. Recently, excluding PKS 1004+13, the population of FR IIBAL quasars has increased to ten objects [8,25], although some of them still require confirmation. Their symmetric structures indicate an "edge-on" orientation, which in turn supports an alternative hypothesis described as "unification by time", with BAL quasars characterised as young or recently refuelled quasars $[2,7]$. There had been only one attempt (and only at $1.6 \mathrm{GHz}$ with the EVN) to image radio structures of the smallest (and probably the youngest) BAL quasars [11]. In this paper we present high frequency VLBA images of another very compact BAL quasar $-1045+352$.

\section{Observations}

$1045+352$ belongs to the primary sample of 60 candidates for CSS sources selected from the VLA FIRST catalogue [22]. Initial observations of all the candidates were made with MERLIN at $5 \mathrm{GHz}$ [12] and 1.7, 5 and 8.4-GHz VLBA follow-up of $1045+352$ was carried out on 13 November 2004 in a snapshot mode with phase-referencing. The target source scan was interleaved with a scan on a phase reference source and the total cycle time (target and phase-reference) was $\sim 9$ minutes including telescope drive times, with $\sim 7$ minutes actually on the target source per cycle.

The whole data reduction process was carried out using standard AIPS procedures. For the target source and at each frequency, the corresponding phase-reference source was mapped and the phase errors so determined were applied to the target sources, which were then mapped using a few cycles of phase self-calibration and imaging. IMAGR was used to produce the final "naturally weighted" images shown in Fig. 1. 
Table 1: Basic parameters of $1045+352$

\begin{tabular}{lr}
\hline \hline Parameter & Value \\
\hline Source name (B1950) & $1045+352$ \\
Source right ascension (J2000) extracted from FIRST & $10^{\mathrm{h}} 48^{\mathrm{m}} 34.247$ \\
Source declination $(\mathrm{J} 2000)$ extracted from FIRST & $+34^{\circ} 57.24$.' $^{\prime} 9$ \\
Total flux density $S_{1.4 \mathrm{GHz}}(\mathrm{mJy})$ extracted from FIRST & 1051 \\
$\log P_{1.4 \mathrm{GHz}}\left(\mathrm{W} \mathrm{Hz}{ }^{-1}\right)$ & 27.65 \\
Total flux density $S_{4.85 \mathrm{GHz}}(\mathrm{mJy})$ extracted from GB6 & 439 \\
Spectral index $\alpha_{1.4 \mathrm{GHz}}^{4.85 \mathrm{Gz}}$ & -0.70 \\
Largest Angular Size measured in the 5-GHz MERLIN image & $0 .{ }^{\prime \prime} 5$ \\
Largest Linear Size $\left(H_{0}=100 \mathrm{~km} \mathrm{~s}^{-1} \mathrm{Mpc}^{-1}, q_{0}=0.5\right)\left(h^{-1} \mathrm{kpc}\right)$ & 2.1 \\
\hline
\end{tabular}

\section{Results and discussion}

According to SDSS/DR5, $1045+352$ is a galaxy at $\mathrm{RA}=10^{\mathrm{h}} 48^{\mathrm{m}} 34^{\mathrm{s}} .242$, Dec $=+34^{\mathrm{o}} 57$ ! 24 . $^{\prime} 95$, which is marked with a cross in the MERLIN map (Fig. 1). However, the spectral observations [23] have shown $1045+352$ to be a quasar with a redshift of $z=1.604$. It has also been classified as a HiBAL QSO based upon the observed very broad CIV absorption, and is a very luminous submillimetre object with detections at both $850 \mu \mathrm{m}$ and $450 \mu \mathrm{m}$ [23].

Our MERLIN and VLBA maps of 1045+352 (Fig. 1) show this source to be extended in both $\mathrm{NE} / \mathrm{SW}$ and NW/SE directions. The central compact feature with a steep spectrum visible in all the maps is probably a radio core. The VLBA image at $1.7 \mathrm{GHz}$ shows radio structure extended in a NE/SW direction, with the SW jet being weaker than that in the NE. The NE/SW emission is visible in the 5-GHz MERLIN image as a few compact features. The 5-GHz VLBA image shows also a core and a one-sided jet pointing to the East. The radio structure in the 8.4-GHz VLBA image is similar to that at $5 \mathrm{GHz}$ : an extended radio core and a jet pointing in an easterly direction.

The observed radio morphology of 1045+352 could indicate a restart of activity accompanied by a reorientation of the jet axis with the NE/SW radio emission being the first phase of activity, now fading away, and the extension in the NW/SE direction being a signature of the current phase of activity.

A reorientation of the jet axis may result from a merger with another black hole. It has been shown [16] that a rapid change of jet orientation can be caused by even a minor merger because of a spin-flip of the central active black hole arising from the coalescence of inclined binary black holes. Also, the Bardeen-Petterson effect [1] can cause a realignment of a rotating supermassive black hole (SMBH) and a misaligned accretion disk [14], the timescale of such a realignment being $t<10^{5}$ years. The interaction/realignment of a binary and its accretion disk leads to the development of X-shaped sources [14]. 1045+352 is not a typical X-shaped source, however, the realignment of a rotating SMBH followed by a repositioning of the accretion disk and jets is a plausible interpretation for misaligned radio structures, even if they are not conspicuously $\mathrm{X}$-shaped [5].

The radio luminosity of $1045+352$ at $1.4 \mathrm{GHz}$ (Table 1 ) is high, making this source one of the most radio-luminous BAL quasars, with a value similar to that of the first known radio-loud 

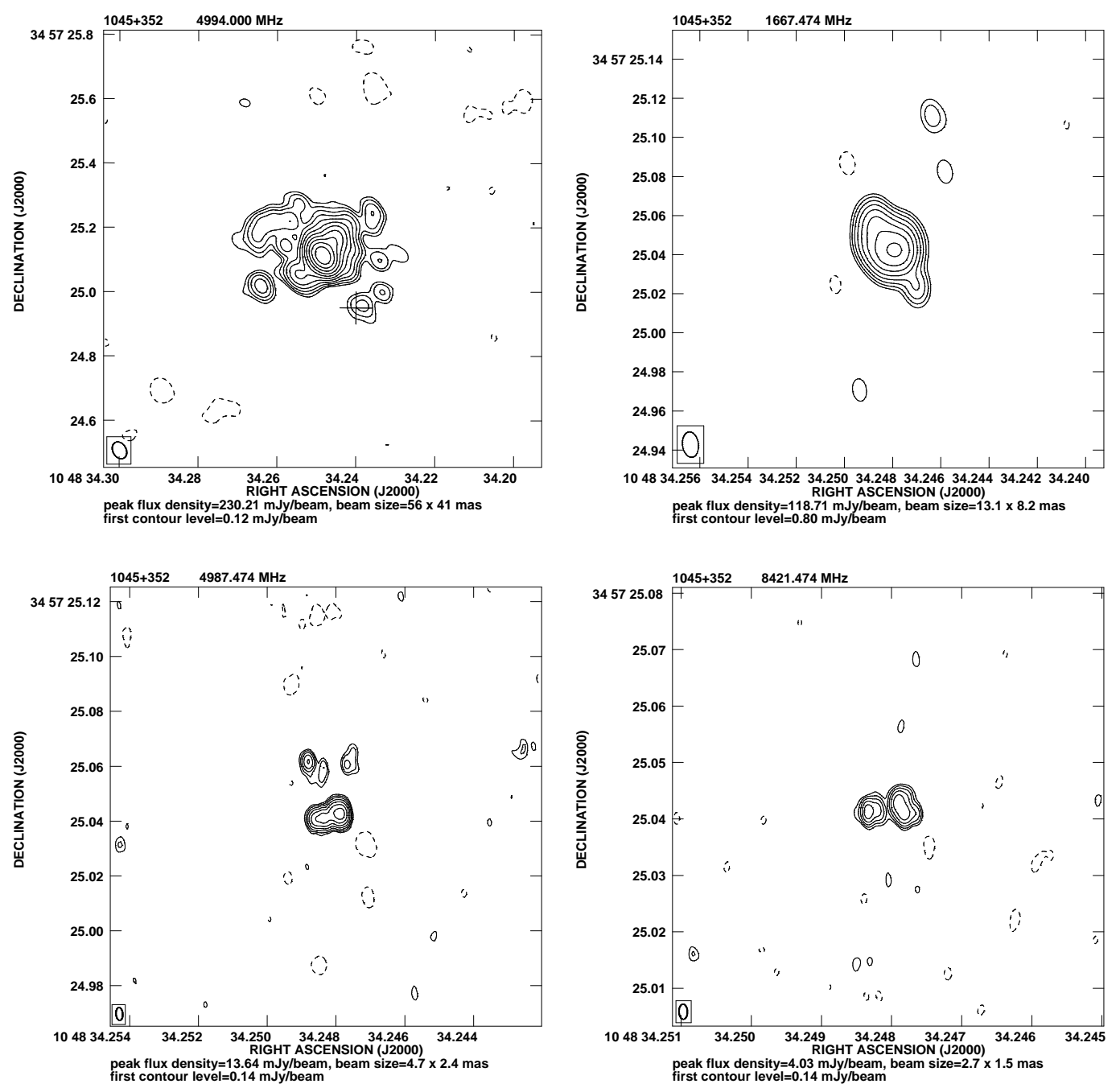

Figure 1: The MERLIN 5-GHz (upper left) and VLBA 1.7, 5 and 8.4-GHz maps of 1045+352. Contours increase by a factor 2 and the first contour level corresponds to $\approx 3 \sigma$. Cross indicates the position of an optical object found using the SDSS/DR5.

BAL QSO with an FR II structure, FIRST J101614.3+520916 [7]. The radio-loudness parameter, $R^{*}$, defined as the $K$-corrected ratio of the 5-GHz radio flux to $2500 \AA$ optical flux [20] has been calculated for $1045+352$ and amounts to $\log \left(R^{*}\right)=4.9$. For this, a global radio spectral index, $\alpha_{\text {radio }}=-0.8$ and an optical spectral index, $\alpha_{\text {opt }}=-1.0$, have been assumed and the SDSS $g^{\prime}$ magnitude has been converted to the Johnson-Morgan-Cousins $B$ magnitude using the transformation formula [19]. Corrections have also been made for intrinsic extinction (local to the quasar) calculated assuming a Milky-Way extinction curve [23]. Even after correction, $\log \left(R^{*}\right)>1$ and amounts to $\log \left(R^{*}\right)=4.1$, which means that $1045+352$ is still radio-loud object. The two-sided asymmetric structure of $1045+352$ can indicate the source grows up in an asymmetric environment which is very typical among small scale CSS sources [18]. However, it was also found $[18,10]$ that the radio properties of CSS sources are consistent with the unified scheme in which the axes of the quasars 
are observed close to the line of sight. It is then likely that in the case of the BAL quasar $1045+352$ the orientation effect is substantial.

\section{Conclusions}

$1045+352$ is a very radio-luminous BAL quasar, whose complex structure is suggestive of restarted activity and may have resulted even from a minor merger. The radio morphology of $1045+352$ shows also that the radio jets and BALs can coexist, at least during some stage of the quasar lifetime. According to [13], in radio-loud systems, the jets then remove the clouds responsible for the generation of BALs, so the extended radio structures showing BAL features are very rare.

$1045+352$ is also a CSS object. CSSs constitute an intermediate class in an evolutionary sequence of radio sources between smaller GPS objects and very extended Large Symmetric Objects (LSO). They are young sources with a typical age of $\sim 10^{5}$ years [17], which is an argument in favour of an evolutionary scenario for BAL quasars, in which the BAL features appear at a very early stage in the quasar evolution.

\section{Acknowledgement}

This work was supported by Polish Ministry of Education and Science under grant 1 P03D 00830 .

\section{References}

[1] Bardeen, J. M. \& Petterson, J. A., The Lense-Thirring Effect and Accretion Disks around Kerr Black Holes, 1975, ApJ, 195, L65

[2] Becker, R. H., White, R. L., Gregg, M. D., et al., Properties of Radio-selected Broad Absorption Line Quasars from the First Bright Quasar Survey, 2000, ApJ, 538, 72

[3] Brotherton, M. S., van Breugel, W., Smith, R. J., et al., Discovery of Radio-Loud Broad Absorption Line Quasars Using Ultraviolet Excess and Deep Radio Selection, 1998, ApJ, 505, L7

[4] Brotherton, M. S., Croom, S. M., De Breuck, C., Becker, R. H. \& Gregg, M. D., The Twice-Overlooked, Second Fanaroff-Riley II Broad Absorption Line Quasar LBQS 1138-0126, 2002, AJ, 124, 2575

[5] Cohen, A. S., Clarke, T. E., Ferretti, L., \& Kassim, N. E., Resolving the Steep-Spectrum Emission in the Central Radio Source in $\mathrm{ZwCl} 0735.7+7421,2005$, ApJ, 620, L5

[6] Gopal-Krishna \& Wiita, P. J., Extragalactic radio sources with hybrid morphology: implications for the Fanaroff-Riley dichotomy, 2000, A\&A, 363, 507

[7] Gregg, M. D., Becker, R. H., Brotherton, M. S., et al., Discovery of a Classic FR II Broad Absorption Line Quasar from the FIRST Survey, 2000, ApJ, 544, 142

[8] Gregg, M. D., Becker, R. H. \& de Vries, W., FR II Broad Absorption Line Quasars and the Life Cycle of Quasars, 2006, ApJ, 641, 210

[9] Hewett, P. C. \& Foltz, C. B., The Frequency and Radio Properties of Broad Absorption Line Quasars, 2003, AJ, 125, 1784 
[10] Jeyakumar, S., Wiita, P. J., Saikia, D. J. \& Hooda, J. S., Jet propagation and the asymmetries of CSS radio sources, 2005, A\&A, 432, 823

[11] Jiang, D. R. \& Wang, T. G., EVN observation of three Broad Absorption Line quasars, 2003, A\&A, 397, L13

[12] Kunert, M., Marecki, A., Spencer, R. E., Kus, A. J., \& Niezgoda J., FIRST-based survey of Compact Steep Spectrum sources. I. MERLIN images of arc-second scale objects, 2002, A\&A, 391, 47

[13] Lipari, S. L. \& Terlevich, R. J., Evolutionary unification in composite active galactic nuclei, 2006, MNRAS, 368, 1001

[14] Liu, F. K., X-shaped radio galaxies as observational evidence for the interaction of supermassive binary black holes and accretion disc at parsec scale, 2004, MNRAS, 347, 1357

[15] Menou, K., Vanden Berk, D. E., \& Ivezić, Ž., Broad Absorption Line Quasars in the Sloan Digital Sky Survey with VLA FIRST Radio Detections, 2001, ApJ, 561, 645

[16] Merritt, D. \& Ekers, R. D., Tracing Black Hole Mergers Through Radio Lobe Morphology, 2002, Science, 297, 1310

[17] Murgia, M., Fanti, C., Fanti, R., et al., Synchrotron spectra and ages of compact steep spectrum radio sources, 1999, A\&A, 345, 769

[18] Saikia, D. J., Jeyakumar, S., Salter, C. J., et al., Compact steep-spectrum sources from the S4 sample, 2001, MNRAS, 321, 37

[19] Smith, J. A., Tucker, D. L., Kent, S., et al., The u'g'r'i'z' Standard-Star System, 2002, AJ, 123, 2121

[20] Stocke, J. T., Morris, S. L., Weymann, J. T. \& Foltz, C. B., The radio properties of the broad-absorption-line QSOs, 1992, ApJ, 396, 487

[21] Weymann, R. J., Morris, S. L., Foltz, C. B., \& Hewett, P. C., Comparisons of the emission-line and continuum properties of broad absorption line and normal quasi-stellar objects, 1991, ApJ, 373, 23

[22] White, R. L., Becker, R. H., Helfand, D. J., \& Gregg, M. D., A Catalog of 1.4 GHz Radio Sources from the FIRST Survey, 1997, ApJ, 475, 479

[23] Willott, C. J., Rawlings, S., Archibald, E. N., \& Dunlop, J. S., A submillimetre difference between radio galaxies and radio quasars: evidence for quasar-heated dust and synchronized submillimetre and radio source activity, 2002, MNRAS, 331, 435

[24] Wills, B. J., Brandt, W. N., \& Laor, A., PKS 1004+13: A High-Inclination, Highly Absorbed Radio-loud QSO-the First Radio-loud Broad Absorption Line QSO at Low Redshift?, 1999, ApJ, 520, L91

[25] Zhou, H., Wang, T., Wang, H., et al., Polar Outflows in Six Broad Absorption Line Quasars, 2006, ApJ, 639, 716 